\title{
Uncertain Data Privacy Protection Based on K-anonymity Via Anatomy
}

\author{
Ren Xiangmin ${ }^{1,2, a}$ Yang Jing ${ }^{1, b}$ Zhang Jianpei $^{1, c}$ Jia Zongfu $^{2, d}$ \\ ${ }^{1}$ College of Computer Science and Technology, Harbin Engineering University, Harbin, China \\ ${ }^{2}$ School of Software, Harbin University, Harbin, China \\ amin0070@sina.com, byangjing@hrbeu.edu.cn, 'zhangjianpei@hrbeu.edu.cn, dsjjzf@hrbu.edu.cn
}

Keywords: k-anonymity, uncertain data, privacy protection, UADK-anonymity

\begin{abstract}
In traditional database domain, $k$-anonymity is a hotspot in data publishing for privacy protection. In this paper, we study how to use $k$-anonymity in uncertain data set, use influence matrix of background knowledge to describe the influence degree of sensitive attribute produced by QI attributes and sensitive attribute itself, use $\mathrm{BK}(\mathrm{L}, \mathrm{K})$-clustering to present equivalent class with diversity, and a novel UDAK-anonymity model via anatomy is proposed for relational uncertain data. We will extend our ideas for handling how to solve privacy information leakage problem by using UDAK-anonymity algorithms in another paper.
\end{abstract}

\section{Introduction}

With the rising of data mining technology and the appearances of data stream and uncertain data technology etc, individual data, the enterprise data are possibly leaked at any moments, so the data security has become nowadays the main topic of information security. With the development of Sensor network, Web service and RFID in recent years, uncertain data has become ubiquitous in economy, military, logistics, finance, telecommunication areas and so on. Uncertain data management and privacy protection have become an important research direction and a hot area of research[1].

$K$-anonymity [2], a model put forward by Samarati P and Sweeney L in 1998 to avoid privacy leaks, requests existence of a certain amount of unrecognizable individuals in the publicized data which make the aggressor disable to distinguish the concrete individual of privacy, and prevent the leak of individual privacy. $K$-anonymity got the universal concern of the academic circles, and a lot of scholars research and develop the technology on different levels. But it was a $k$-anonymity privacy protection model of deterministic data, currently, research in uncertain data publishing based on $k$-anonymity is limited, it needs a new model to represent the $k$-anonymity privacy protection of uncertain data.

Charu C. Aggarwal [3] presents an uncertain version of the $k$-anonymity model, which has the additional feature of introducing greater uncertainty for the adversary over an equivalent deterministic model. He tests the effectiveness of the privacy transformation on the problems of query estimation and classification, and show that the technique retains greater accuracy than other $k$-anonymity models. Wu jiawei, et al. explore several new modeling methods[4]. A model space which consists of $\mathrm{K}_{\text {attr }}, \mathrm{K}_{\text {tuple }}, \mathrm{K}_{\text {upperlower }}$ and $\mathrm{K}_{\text {tree }}$ model is built, the $\mathrm{K}_{\text {attr }}$ model uses the attribute-ors ways to describe the uncertainty in the quasi-identifier attribute $(Q I)$ values of the $k$-anonymity privacy protection model, the $\mathrm{K}_{\text {tuple }}$ model takes $Q I$ values as relations and use the tuple-ors ways to describe the relations. The completeness and closure about these models are discussed.

This paper explores a new $k$-anonymity privacy protection model for relational uncertainty data by anatomy. 


\section{Related concepts}

\section{k-anonymity}

\section{Definition 1: $\boldsymbol{k}$-anonymity}

Let $R T\left(A_{1}, \ldots, A_{n}\right)$ be a table and $Q I_{R T}$ be the quasi-identifier associated with it. $R T$ is said to satisfy $k$-anonymity if and only if each sequence of values in $R T\left[Q I_{R T}\right]$ appears with at least $k$ occurrences in $R T\left[Q I_{R T}\right][5]$.

Table 1 is an example of $k$-anonymity, sensitive attribute $(S I)$ is Disease, $Q I_{T}=\{$ Race, Education, Age, Sex, $Z I P\}$ and $k=2$. In particular, $t 1\left[Q I_{T}\right]=t 2\left[Q I_{T}\right], t 3\left[Q I_{T}\right]=t 4\left[Q I_{T}\right], t 5\left[Q I_{T}\right]=t 6\left[Q I_{T}\right]=t 7\left[Q I_{T}\right]$, $t 8\left[Q I_{T}\right]=t 9\left[Q I_{T}\right]$.

Table 1 Example of $k$-anonymity, where $k=2$ and $Q I=\{$ Education, Age, Sex, ZIP $\}$

\begin{tabular}{|c|l|c|c|c|l|}
\hline Num & Education & Age & Sex & ZIP & \multicolumn{1}{|c|}{ Disease } \\
\hline t1 & Master & $(15,40]$ & $\mathrm{F}$ & $3115^{* *}$ & flu \\
\hline t2 & Master & $(15,40]$ & $\mathrm{F}$ & $3115^{* *}$ & lung cancer \\
\hline t3 & Master & $(40,60]$ & $\mathrm{M}$ & $3114^{* *}$ & lung cancer \\
\hline t4 & Master & $(40,60]$ & $\mathrm{M}$ & $3114^{* *}$ & lung cancer \\
\hline t5 & Bachelor & $(40,60]$ & $\mathrm{F}$ & $3114^{* *}$ & lung cancer \\
\hline t6 & Bachelor & $(40,60]$ & $\mathrm{F}$ & $3114^{* *}$ & short breath \\
\hline t7 & Bachelor & $(40,60]$ & $\mathrm{F}$ & $3114^{* *}$ & obesity \\
\hline t8 & Ph.D & $(40,60]$ & Person & $3114^{* *}$ & mammary cancer \\
\hline t9 & Ph.D & $(40,60]$ & Person & $3114^{* *}$ & mammary cancer \\
\hline
\end{tabular}

Generalization. Given an attribute $A$, a generalization for an attribute is a function on $A$. That is, each $f: A \rightarrow B$ is a generalization, it also says that: ${ }^{A_{0}} \stackrel{f_{0}}{\longrightarrow} A_{l}{ }_{i l} \longrightarrow \cdots \stackrel{f_{n-1}}{\longrightarrow} A_{n}$ is a generalization sequence or a functional generalization sequence [6]. Fig.1 provides an example of generalization hierarchies.

Any

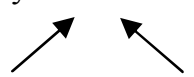

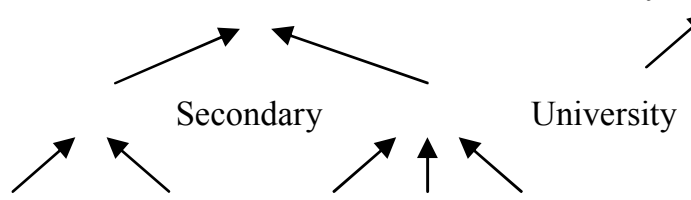

Primary Sch. Second Sch. Bachelor Master Ph.D

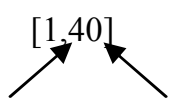

$(40,80]$
$[1,110]$

Education

Age

$(40,110]$

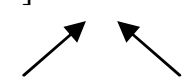

Person

Fig.1 Gerneralization Hierarchies of $\{$ Education, Age, Sex $\}$

Influence matrix based on background knowledge. Background knowledge describes the influence of a variety of SI produced by $Q I[7,8]$. Background knowledge can be acquired from domain expert, and also can be acquired by analyzing basic data directly.

We use relation and sensitive degree matrix $\mathrm{m} \mid \mathrm{s}$ to describe the influence degree of SI produced by $Q I$ and $S I$ itself, introducing notation as follows:

$t_{i j}:$ the influence degree of NO. $j$ SI produced by NO. $i Q I$.

$b_{i}$ : the weight of $S I$ value of NO. $i$.

Influence matrix $\mathrm{M} \mid \mathrm{s}$ is with $m$ rows and $n+1$ columns, $m$ is the number of $S I, n$ is the number of $Q I$ attribute, then the matrix is as follows:

$$
\mathrm{M} \mid \mathrm{S}=\left(\mathrm{t}_{\mathrm{ij}} \mid \mathrm{b}_{\mathrm{i}}\right)_{\mathrm{m} \times(\mathrm{n}+1)}=\left[\begin{array}{cccccc|c}
\mathrm{QI}_{1} & \mathrm{QI}_{2} & \mathrm{QI}_{3} & \mathrm{QI}_{4} & \cdots & \mathrm{QI}_{\mathrm{n}} & \mathrm{S} \\
\mathrm{t}_{11} & \mathrm{t}_{12} & \mathrm{t}_{13} & \mathrm{t}_{14} & \cdots & \mathrm{t}_{\mathrm{ln}} & \mathrm{b}_{1} \\
\cdots & \cdots & \cdots & \cdots & \cdots & \cdots & \cdots \\
\mathrm{t}_{11} & \mathrm{t}_{\mathrm{i}} & \mathrm{t}_{\mathrm{i}} & \mathrm{t}_{4} & \cdots & \mathrm{t}_{\mathrm{in}} & \mathrm{b}_{\mathrm{i}} \\
\cdots & \cdots & \cdots & \cdots & \cdots & \cdots & \cdots \\
\mathrm{t}_{\mathrm{m} 1} & \mathrm{t}_{\mathrm{m} 2} & \mathrm{t}_{\mathrm{m} 3} & \mathrm{t}_{\mathrm{m}} & \cdots & \mathrm{t}_{\mathrm{mn}} & \mathrm{b}_{\mathrm{m}}
\end{array}\right]
$$


The weight value of $t_{i j}$ and $b_{i}$ is specified by expert or experience value, for example, we can divide weight of $Q I$ in Table 1 into 5 grades, 1,0.8,0.4,0.1,0, and divide weight of $S$ in Table 1 into 5 grades, $0.10,0.30,0.50,0.80,0.90$. The $f l u$ is common ailments, disease weight can use 0.11 , because of the characteristic of local outbreaks of $f l u, Z I P$ weight use 0.8, Sex weight use 0.2 etc. The disease weight of obesity can use 0.12 , the disease weight of flu and obesity are all $0.1,0.01$ and 0.02 denotes different ailment. The disease weight of short breath is 0.31 , the major diseases weight of lung cancer, mammary cancer and AIDS use 0.91, 0.92 and 0.93, different disease must have different disease weight value. Then the relation and sensitive degree matrix based on Table 1 is as follows:

$$
\mathrm{M} \mid \mathrm{S}=\left(\mathrm{t}_{\mathrm{ij}} \mid \mathrm{b}_{\mathrm{i}}\right)_{9 \times(5+1)}=\left[\begin{array}{ccccc|c}
\text { Race } & \text { Education } & \text { Age } & \text { Sex } & \text { ZIP } & \text { Disease } \\
0 & 0 & 0 & 0.2 & 0.8 & 0.11 \\
\ldots & \ldots & \ldots & \ldots & \ldots & \ldots \\
0 & 0 & 0.4 & 1 & 0 & 0.92 \\
0 & 0 & 0.4 & 1 & 0 & 0.92
\end{array}\right]
$$

Anatomy. Anatomy was proposed by Xiaokui Xiao et al, it means $Q I$ and $S I$ published in different table, instead of publishing one single table with the generalized values, $Q I$ table included a unique identifier: equivalent class(QI-group) ID, SI table included equivalent class ID too, $S I$ of each QI-group, and count. Anatomy overcomes the drawbacks of generalization. Extensive experiments confirm that anatomy permits researchers to derive from the published tables, highly accurate aggregate information about the unknown microdata, with an average error below 10\% [9]. For example, table 3 satisfied 3-diversity anatomy table according to table 2 by anatomy. In paper [10], they proved that the resulting published tables $\operatorname{NSS}(Q I)$ and $\operatorname{SS}(S I)$ satisfy $p$-sensitive $k$-anonymity property, that is to say, anatomy satisfy $p$-sensitive $k$-anonymity property.

Table 2 The original data table

\begin{tabular}{|c|c|c|}
\hline Sex & ZIP & Disease \\
\hline F & 311578 & flu \\
\hline F & 311579 & chest pain \\
\hline F & 311579 & hypertension \\
\hline M & 311581 & obsity \\
\hline M & 311582 & short breath \\
\hline M & 311582 & hypertension \\
\hline F & 311588 & obesity \\
\hline F & 311588 & chest pain \\
\hline
\end{tabular}

Table 3 The 3-diversity data table by anatomy

\begin{tabular}{|c|c|c|}
\hline \multicolumn{3}{|c|}{ QI attribute } \\
\hline Sex & ZIP & ID \\
\hline F & 311578 & 1 \\
\hline F & 311579 & 1 \\
\hline M & 311579 & 1 \\
\hline M & 311581 & 2 \\
\hline M & 311582 & 2 \\
\hline F & 311582 & 2 \\
\hline F & 311588 & 3 \\
\hline F & 311588 & 3 \\
\hline F & 311589 & 3 \\
\hline
\end{tabular}

\begin{tabular}{|c|c|}
\hline \multicolumn{2}{|c|}{ Sensitive attrbute } \\
\hline ID & Disease \\
\hline \multirow{3}{*}{1} & flu \\
\cline { 2 - 2 } & chest pain \\
\cline { 2 - 2 } & hypertension \\
\cline { 2 - 2 } 2 & obsity \\
\cline { 2 - 2 } & short breath \\
\cline { 2 - 2 } & hypertension \\
\cline { 2 - 2 } 3 & obesity \\
\cline { 2 - 2 } & chest pain \\
\hline \multirow{3}{*}{3} & cancer \\
\hline
\end{tabular}

Constructs for Uncertainty. There are two different constructs for u-tuples(uncertain tuples)[11,12]:

attribute-ors: An attribute-or in a u-tuple specifies a set of alternative values for an attribute. For example, $t 1$ contains an attribute-or in its first field and represents one of two possible tuples: (Bachelor, insomnia) or (Master, insomnia). 
Table 4 Uncertain data table with attribute-ors construct

\begin{tabular}{|c|l|l|}
\hline Num & \multicolumn{1}{|c|}{ Education } & \multicolumn{1}{|c|}{ Disease } \\
\hline t1 & \{Bachelor, Master $\}$ & insomnia \\
\hline t2 & Second School & flu \\
\hline t3 & Ph.D & mammary cancer \\
\hline t4 & Master & short breath \\
\hline t5 & Primary School & lung cancer \\
\hline t6 & Ph.D & mammary cancer \\
\hline
\end{tabular}

tuple-ors: A tuple-or in a u-tuple specifies a set of possible tuples. For example, the uncertainty in the previous example can also be represented by:

Table 5 Uncertain data with tuple-ors construct

(Bachelor, insomnia)||(Master, insomnia)

\section{K-anonymity privacy protection model for uncertain data via anatomy}

First, we preprocess the uncertainty data table which makes the uncertainty data table become a deterministic data table, namely, the uncertain data has been generalized, for example, $\{$ Bachelor, Master $\} \rightarrow$ University, then model the data of deterministic data table by $k$-clustering and anatomy. When we create a deterministic data table from an uncertainty data table, each uncertainty QI(attribute-or) is labeled with QIID or TupleID attribute in order to keep the uncertainty of QI of original data in uncertainty data table, QIID or TupleID is a appended attribute column, which value represents location in the deterministic data table. At the same time, we divide the uncertainty $S I$ into two or more fields of $S I$ in order to keep the uncertainty of $S I$ of original data in uncertainty data table. That is to say, uncertain data is stored in a relational database, then we can use traditional $k$-anonymity model to represent the privacy protection of uncertain data.

UDAK-anonymity model. UDAK-anonymity model(uncertain data anatomy $k$-anonymity model) is built for attribute-ors construct, modeling process needs three steps: preprocessing, $\mathrm{BK}(\mathrm{L}, \mathrm{K})$-clustering [13], and anatomy.

\section{Preprocessing}

1. Create deterministic table by generalization and partition

Definition 2. Deterministic uncertian data table. $T\left(A_{1}, \ldots, A_{n}\right)$ is an uncertain data table, if $T$ can be change into deterministic data table $T^{\prime}$ by generalization and partition, we say $T^{\prime}$ is a deterministic uncertian data table.

Table 6 The original data table including uncertain data with attribute-ors construct

\begin{tabular}{|c|l|c|l|l|}
\hline Num & \multicolumn{1}{|c|}{ Education } & Age & \multicolumn{1}{|c|}{ Sex } & \multicolumn{1}{|c|}{ Disease } \\
\hline t1 & $\{$ Bachelor, Master $\}$ & 25 & M & insomnia \\
\hline t2 & Bachelor & 21 & M & $\{$ obesity, flu $\}$ \\
\hline t3 & Ph.D & 35 & F & mammary cancer \\
\hline t4 & $\{$ Master, Ph.D $\}$ & $\{41,48\}$ & M & $\{$ short breath, obesity \\
\hline t5 & Master l & 45 & M & lung cancer \\
\hline t6 & Ph.D & 36 & F & mammary cancer \\
\hline
\end{tabular}

Generalize QIs which include uncertain data with attribute-ors construct and divide the uncertainty SI into two or more fields of SI. For example, Table 6 is the original data table including uncertain data with attribute-ors construct, Table 7 (deterministic uncertian data table) is the deterministic data table by generalization and partition according to Table 6 . \{Bachelor, Master $\} \rightarrow$ University in $\mathrm{t} 1$, \{obesity, flu $\} \rightarrow \mathrm{t} 2[$ disease 1$]=$ obesity, $t 2[$ disease 2$]=f l u$ in $t 2,\{$ Master, $P h . D\} \rightarrow$ University, $\{41,48\} \rightarrow \max \{41,48\}=48$, \{short breath, obesity $\} \rightarrow t 4$ [disease1] = short breath, $t 4[$ disease $]=$ obesity in $t 4$. In Table $7, t 1[Q I I D]=22$ means the second field(Education) is the generalization value of uncertain data according to generalization hierarchies(Fig.1), and it has two uncertain data(two child nodes), $t 2[Q I I D]=S I 2$ means the uncertainty $S I$ attribute was divided into two fields of $S I$ attribute, $t 2[$ disease 1$]=$ obesity, $t 2[$ disease 2$]=f l u$, so does $t 4$. If $t_{i}[Q I I D]=0$, it represent that $t_{i}$ is a deterministic data. 
Table 7 The deterministic data table by generalization and partition according to Table 6

\begin{tabular}{|c|l|l|c|l|l|l|}
\hline Num & \multicolumn{1}{|c|}{ QIID } & \multicolumn{1}{c|}{ Education } & Age & \multicolumn{1}{c|}{ Sex } & \multicolumn{1}{c|}{ Disease1 } & Disease2 \\
\hline t1 & 22 & University & 25 & M & insomnia & \\
\hline t2 & SI2 & Bachelor & 21 & M & obesity & flu \\
\hline t3 & 0 & Ph.D & 35 & F & mammary cancer & \\
\hline t4 & 2232 SI2 & University & 48 & M & short breath & obesity \\
\hline t5 & 0 & Master & 45 & M & lung cancer & \\
\hline t6 & 0 & Ph.D & 36 & F & mammary cancer & \\
\hline
\end{tabular}

2. Create influence matrix based on background knowledge according to section 2.3

BK(L,K)-clustering

Definition 3. K-Clustering. $S$ is a data set which has $n$ tuples, $K$ is anonymous parameter, K-Clustering is Clustering set: $\varepsilon=\left\{e_{1}, \ldots, e_{m}\right\}$ which satisfies the following conditions:

(1) $\forall i \neq j \in\{1, \ldots m\}, e_{i} \cap e_{j}=\varnothing$;(2) $\bigcup_{i=1, \ldots, m} e_{i}=S$;(3) $\forall e_{i} \in \varepsilon,\left|e_{i}\right| \geq K$

(4) $\frac{1}{m} \sum_{l=1, \ldots, m}\left|e_{l}\right| * M A X_{i, j=1, \ldots, \mid}\left|e_{\mid}\right| \Delta(t(l, i), t(l, j))$ is minimum

Here $\left|e_{l}\right|$ is the amounts of tuples in clustering $e_{l}, t(l, i)$ is NO. $i$ tuple in clustering $e_{l}$, $\Delta(t(l, i), t(l, j))$ is the distance between NO. $i$ tuple and NO. $j$ tuple, $M A X_{i, j=1, \ldots,|e l|} \Delta(t(l, i), t(l, j))$ is maximum distance in clustering $e_{l}$ [13].

Definition 4. BK(L,K)-clustering $((\mathrm{L}, \mathrm{K})$-Clustering based on influence matrix of background knowledge). $T\left(A_{1}, \ldots, A_{n}\right)$ is a table, if $T$ satisfies $\mathrm{K}$-Clustering, and satisfies the following conditions:

(1) $\forall b_{i}<c$ in clustering $e_{m}$, all tuples in $e_{m}$ should be anatomized directly. Otherwise must satisfy condition (2). Here, threshold $c>0, b_{i}$ is $S$ column vector in influence matrix $\mathrm{m}|\mathrm{s}, 1 \leq i \leq| e_{m}|,| e_{m} \mid$ is the amounts of tuples in clustering $e_{m}$.

(2) $L=\sum_{j=1, \ldots,|e m|} \operatorname{count}\left(\left|b_{i}-b_{i}\right|>0\right), 1 \leq i \leq\left|e_{n}\right|, b_{i}, b_{j}$ is $S$ column vector in influence matrix $\mathrm{M} \mid \mathrm{s}, L$ is the amounts of different sensitive attribute value, and $L$ makes sensitive attribute diversity, otherwise further improve the generalization or suppression.

We say $\mathrm{T}$ satisfies $\mathrm{BK}(\mathrm{L}, \mathrm{K})$-clustering.

\section{BK(L,K)-anonymity with anatomy}

Definition 5. BK(L,K)-anonymity with anatomy. ((L,K)-anonymity with anatomy based on influence matrix of background knowledge). $T\left(A_{1}, \ldots, A_{n}\right)$ is table, if $T$ satisfies BK(L,K)-clustering, then we divided $T$ into $Q I$ table(QIT) and $S I$ table(ST). Specifically, the QIT includes all its exact $Q I$ values, together with its group membership in a new column Group-ID. However, QIT does not store any SI values, ST retains SI statistics of each QI-group, Group-ID and count.

Definition 6. UDAK-anonymity. $T\left(A_{1}, \ldots, A_{n}\right)$ is an uncertain data table, $T$ ' is a deterministic uncertian data table from $T$, and satisfies $\mathrm{BK}(\mathrm{L}, \mathrm{K})$-anonymity with anatomy, we say $T$ ' satisies UDAK-anonymity.

For instance, Table 8 which were anatomized according to table 7 satisfied UDAK-anonymity.

Table 8 The anatomized tables according to table 7

\begin{tabular}{|c|l|l|c|l|l|}
\hline \multicolumn{7}{|c|}{ QIT } \\
\hline Num & QIID & Education & Age & Sex & ID \\
\hline t1 & 22 & University & 25 & M & 1 \\
\hline t2 & 0 & Bachelor & 21 & M & 1 \\
\hline t3 & 0 & $*$ & $*$ & $*$ & 2 \\
\hline t6 & 0 & $*$ & $*$ & $*$ & 2 \\
\hline t4 & 2232 & University & 48 & M & 3 \\
\hline t5 & 0 & Master & 45 & M & 3 \\
\hline
\end{tabular}

\begin{tabular}{|c|l|l|c|}
\hline \multicolumn{4}{|c|}{ ST } \\
\hline ID & \multicolumn{1}{|c|}{ Disease 1 } & Disease 2 & \\
\hline 1 & insomnia & & 1 \\
\hline 1 & obesity & flu & 1 \\
\hline 2 & $\begin{array}{l}\text { mammary } \\
\text { cancer }\end{array}$ & 2 \\
\hline 2 & $\begin{array}{l}\text { mammary } \\
\text { cancer }\end{array}$ & & 1 \\
\hline 3 & short breath & obesity & 1 \\
\hline 3 & lung cancer & & 1 \\
\hline
\end{tabular}

Similarly, we can use UDAK-anonymity model to deal with tuple-ors construct of uncertainty, owing to the limitation of the scope, I won't discuss it in this post. 


\section{Conclusion}

This paper proposed specific modeling method of k-anonymity privacy protection of uncertain data via anatomy, and presented new models of k-anonymity privacy protection, UDAK-anonymity. UDAK-anonymity model not only kept the characteristic of uncertian data, but also provided more useful information for the user, improved the utility of uncertain data. Owing to the limitation of the scope, we will extend our ideas for handling how to solve privacy information leakage problem by using UDAK-anonymity algorithms in another paper.

\section{Acknowledgement}

The authors want to thank the helpful comments and suggestions from the anonymous reviewers. This work was supported by the National Natural Science Foundation of China (Grant No. 61073043), the Natural Science Foundation of Heilongjiang Province of China (Grant No. F200901).

\section{References}

[1] ZHOU Aoying et al. A Survey on the M anagement of Uncertain Data, Chinese journal of Computers. 32(2009) 1-15.

[2] Samarati P, Sweeney L, Generalizing data to provide anonymity when disclosing information, Proceedings of the Seventeenth ACM SIGACT-SIGMOD-SIGART Symposium on Principles of Database Systems, PODS, (1998) 188.

[3] Aggarwal Charu.C, On Unifying Privacy and Uncertain Data Models, Proceedings of the 2008 IEEE 24th International Conference on Data Engineering. (2008) 386-395.

[4] Wu Jia-wei, Liu Guo-hua, Wang Mei,Modeling the Uncertain data in data in the K-anonymity Privacy Protection Mode., CPMPUTER EINGINEERING \& SCIENCE. 33(2011) 7-13.

[5] L. Sweeney, k-anonymity: a model for protecting privacy, International Journal on Uncertainty,Fuzziness and Knowledge-based Systems, 10 (2002) 557-570.

[6] L. Sweeney, Achieving k-anonymity privacy protection using generalization and suppression, International Journal on Uncertainty, Fuzziness and Knowledge-based Systems, 10 (2002) $571-588$.

[7] Xiangmin, Ren, et al., "Research on $\mathrm{CBK}(\mathrm{L}, \mathrm{K})$-anonymity algorithm", International Journal of Advancements in Computing Technology.3(2011) 165-173.

[8] LI Tai-yong, et al., "k-Anonymity via Twice Clustering for Privacy Preservation", Journal of Jilin University. 27(2009) 173-178.

[9] XiaoXiaokui, TaoYufei, Anatomy: Sample and effective protect preservation, Proceedings of the 32nd International Conference on VeryLarge DataBases. (2006)139-150.

[10]Xiaoxun Sun, Hua Wang, Jiuyong Li, David Ross, Achieving p-Sensitive k-Anonymity via Anatomy, Proceedings of the 2009 IEEE International Conference on e-Business Engineering. (2009) 199-205.

[11]Anish Das Sarma, Shubha U. Nabar, Jennifer Widom, Representing Uncertain Data: Uniqueness, Equivalence, Minimization, and Approximation.. Technical Report, Stanford Infolab (2005).

[12]Anish Das Sarma, Omar Benjelloun, Alon Halevy, Shubha Nabar, JenniferWidom, Representing Uncertain Data: Models, Properties, and Algorithms. The VLDB Joural. 18(2009) 989-1019.

[13]Ren Xiangmin, Yang Jing, Zhang Jianpei, Wang Kechao, " Research on CBK(L,K)-Anonymity Algorithm ", International Journal of Advancements in Computing Technology. 3(2011) 165 $-173$. 